\title{
F-Cphl represents a new homing endonuclease family using the Endo VII catalytic motif
}

\author{
Xiaoting Fang ${ }^{1}$, YongLiang Jiang ${ }^{2}$, Kim Li ${ }^{1}$ and Qinglu Zeng ${ }^{1,3,4^{*}}$
}

\begin{abstract}
Background: There are six known families of homing endonucleases, LAGLIDADG, GIY-YIG, HNH, His-Cys box, PD-(D/E)-XK, and EDxHD, which are characterized by their conserved residues. Previously, we discovered a novel homing endonuclease F-Cphl encoded by ORF177 of cyanophage S-PM2. F-Cphl does not resemble any characterized homing endonucleases. Instead, the C-terminus of F-Cphl aligns well with the N-terminal catalytic domain of a Holliday junction DNA resolvase, phage T4 endonuclease VII (Endo VII).

Results: A PSI-BLAST search resulted in a total of 313 Endo VII motif-containing sequences in sequenced genomes. Multiple sequence alignment showed that the catalytically important residues of T4 Endo VII were all well conserved in these proteins. Our site-directed mutagenesis studies further confirmed that the catalytically important residues of T4 Endo VII were also essential for F-Cphl activity, and thus F-Cphl might use a similar protein fold as Endo VII for DNA cleavage. A phylogenetic tree of the Endo VII motif-containing sequences showed that putative resolvases grouped into one clade while putative homing endonucleases and restriction endonucleases grouped into another clade.
\end{abstract}

Conclusions: Based on the unique conserved residues, we proposed that F-Cphl represents a new homing endonuclease family, which was named the DHHRN family. Our phylogenetic analysis could be used to predict the functions of many previously unknown proteins.

Keywords: Homing endonuclease, Group I intron, F-Cphl, Endonuclease VII, Bacteriophage

\section{Background}

Many group I introns contain open reading frames (ORFs) that encode homing endonucleases. In a process called intron-homing, a homing endonuclease cleaves an intronless allele near the intron insertion site (IIS) and repair of the DNA break using the intron-containing allele as template transfers the intron and the homing endonuclease gene into the intronless allele [1]. Homing endonuclease genes are also found as optional free-standing genes inserted between two conserved genes. They can cleave one of the two conserved genes in genomes lacking the homing endonuclease gene. Analogous to intron-homing,

\footnotetext{
*Correspondence: zeng@ust.hk

'Department of Ocean Science, The Hong Kong University of Science and Technology, Clear Water Bay, Kowloon, Hong Kong, China

${ }^{3}$ Division of Life Science, The Hong Kong University of Science and

Technology, Clear Water Bay, Kowloon, Hong Kong, China

Full list of author information is available at the end of the article
}

repair of the DNA break transfers the homing endonuclease gene to the recipient genome, a process that has been called intronless homing $[2,3]$.

Homing endonucleases have been grouped into six families, LAGLIDADG, GIY-YIG, HNH, His-Cys box, PD-(D/E)-XK, and EDxHD, which were named for the conserved amino acid residues (reviewed by [4]). Crystal structures of the six homing endonuclease families have been determined [5-10]. Based on their structural similarities, it was suggested that the $\mathrm{HNH}$ and His-Cys box families should be combined to a $\beta \beta \alpha$-metal superfamily [11]. The catalytic motifs of PD-(D/E)-XK and EDxHD families were also shown to be related [4].

Previously, we identified a novel homing endonuclease F-CphI [12]. It is encoded by ORF177 of cyanophage S-PM2 (genome accession \# AJ630128.1), which is adjacent to the intron-containing $p s b A$ gene $[13,14]$.

(c) The Author(s). 2018 Open Access This article is distributed under the terms of the Creative Commons Attribution 4.0 International License (http://creativecommons.org/licenses/by/4.0/), which permits unrestricted use, distribution, and reproduction in any medium, provided you give appropriate credit to the original author(s) and the source, provide a link to the Creative Commons license, and indicate if changes were made. The Creative Commons Public Domain Dedication waiver (http://creativecommons.org/publicdomain/zero/1.0/) applies to the data made available in this article, unless otherwise stated. 
While F-CphI has specificity for the homologous intronless $p s b A$ gene of phage S-BM4, the group I intron prevents $\mathrm{F}$-CphI cleavage of the S-PM2 psbA gene [12]. In this novel arrangement, an intron prevents self-cleavage by a free-standing homing endonuclease, which in turn provides the intron with potential to invade intronless genomes. Thus, this process has been named "collaborative homing" [12].

ORF177 was originally annotated as similar to gene 49 of phage T4, which encodes endonuclease VII (Endo VII), a Holliday junction DNA resolvase [13, 14]. A BLAST search using F-CphI as the query sequence did not find any characterized homing endonuclease, but typically many other proteins annotated as "Endo VII", "similar to Endo VII", "resolvase", and "gp 49". Aside from Endo VII and its homologues in T-even phages, the enzymatic activities of these proteins had not been determined. Sequence alignment of F-CphI, Endo VII, and other Endo VII-like proteins showed a region of conserved sequence that included the catalytic domain of T4 Endo VII (Fig. 1a). Moreover, the catalytically important residues of Endo VII were all well conserved in these proteins. In this work, we conducted site directed mutagenesis on these conserved residues in $\mathrm{F}-\mathrm{CphI}$ to determine whether they are functionally important. In addition, phylogenetic analysis allowed us to identify potential homing endonucleases and resolvases among the uncharacterized proteins. F-CphI appears to be the first characterized representative of a new family of homing endonucleases that use the Endo VII catalytic motif.

\section{Results}

Endonuclease assay with recombinant F-Cphl

Previously we have used in vitro expressed F-CphI to map its cleavage site in the $p s b A$ gene of phage S-BM4 [12]. In order to further study the biochemical properties of this novel homing endonuclease, we cloned S-PM2 ORF177 into pBAD/Myc-HisB and induced His-tagged F-CphI expression in Escherichia coli cells. After large amounts of soluble F-CphI were induced, one step purification gave $\sim 64 \%$ purity as observed on SDS-PAGE (Additional file 1: Figure S1A). Purified F-CphI was used to digest plasmid DNA containing the F-CphI recognition site for different times. As soon as F-CphI was added into the reaction, the closed-circular plasmid substrates were nicked into open-circular intermediate products, which were then converted into the final linear products (Fig. 2a). To know whether one DNA strand was preferentially nicked, top strand or bottom strand ${ }^{32} \mathrm{P}$ labeled oligonucleotide duplexes containing the F-CphI recognition site were used as substrates. The top strand cleavage products appeared earlier than the bottom strand cleavage products, indicating that F-CphI preferred to nick the top strand first (Fig. 2b). Similarly, Endo VII [15] and the homing endonucleases I-SceI [16], I-TevI [17], I-TevII [18], and I-BmoI [19] also preferentially cleave one strand of the DNA substrate.

\section{Optimal conditions for $\mathrm{F}-\mathrm{Cphl}$ endonuclease activity}

In order to determine the optimal conditions for F-CphI endonuclease activity on the plasmid DNA substrate,
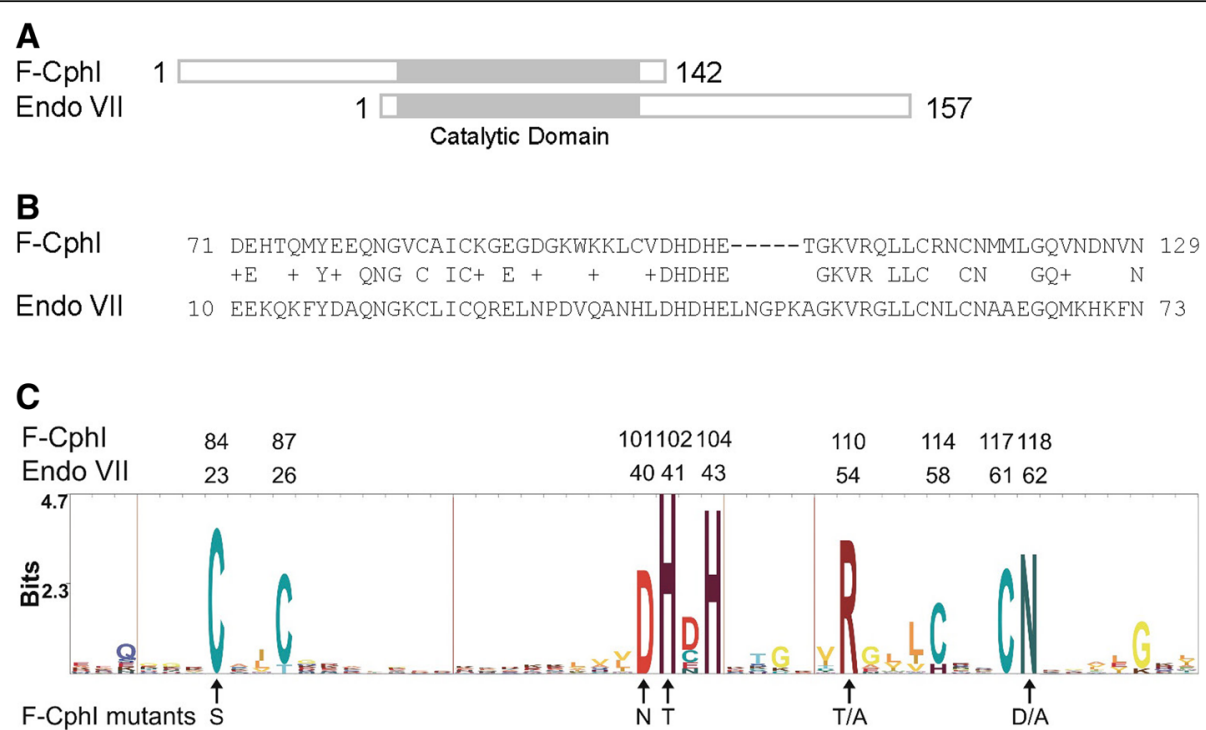

Fig. 1 Sequence alignment. a, b The C-terminal part of F-Cphl aligns with the N-terminal catalytic domain of Endo VII. c Sequence logo shows the consensus sequence from the multiple sequence alignment in Additional file 3: Figure S2. The positions of the conserved residues in F-Cphl and Endo VII are shown on the top, and F-Cphl mutants generated in this study are shown on the bottom. At each position of the sequence logo, the total height of a stack of letters shows the information content in bits that is calculated from a profile hidden Markov model (see Methods), and the height of a letter relative to the total height of letters at a position represents the letter's frequency. The red lines indicate gaps in the multiple sequence alignment 


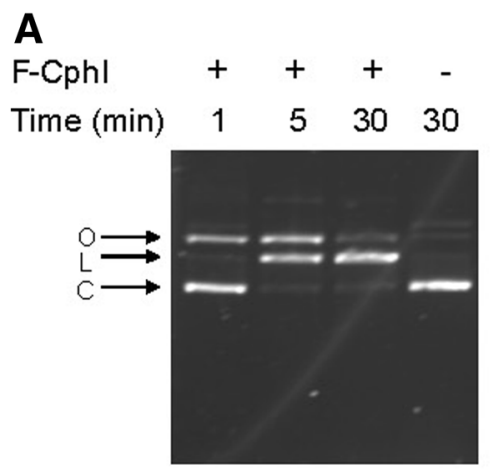

B

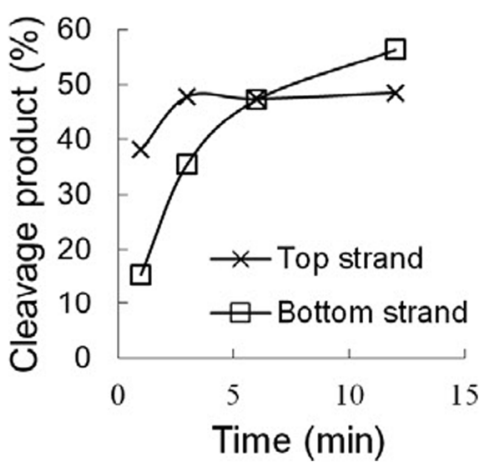

Fig. 2 Strand preference of F-Cphl. a Purified F-Cphl (1600 nM) was used to digest plasmid DNA (5 nM) in the standard endonuclease assay buffer for different times. Closed-circular plasmid DNA (C) was nicked to form open circular DNA $(\mathrm{O})$ and then nicked again on the other strand to form a linear product (L). b Time course activity assay on ${ }^{32}$ P-labeled oligonucleotide duplex. Oligonucleotides SBM4-60Top and SBM4-60Top-r were used to create a $60 \mathrm{bp}$ duplex containing the F-Cphl recognition site from the cyanophage S-BM4 psbA gene. The duplex was labeled on the top or the bottom strand by ${ }^{32} \mathrm{P}$. Labeled duplex substrates were digested by F-Cphl and the cleavage products were separated by electrophoresis on a $4 \%$ denaturing polyacrylamide gel. Substrates labeled on the top or the bottom strand were examined separately. Percent cleavage products are shown over time

each parameter in the standard endonuclease assay was changed systematically (Fig. 3). F-CphI was most active at around $20{ }^{\circ} \mathrm{C}$ (Fig. 3a) and pH 7.0 (Fig. 3b), which is in contrast to $37-70{ }^{\circ} \mathrm{C}$ and alkaline $\mathrm{pH}$ for most biochemically characterized homing endonucleases [20] and T4 Endo VII [21]. The optimal temperature of F-CphI is consistent with the temperature of seawater where cyanophage S-PM2 was isolated, which encodes the gene for F-CphI. F-CphI activity showed a dependence on $\mathrm{Mg}^{2+}$, with an optimum of 4-20 mM (Fig. 3c). In addition, $\mathrm{Mg}^{2+}$ could be replaced by $\mathrm{Mn}^{2+}$ and $\mathrm{Co}^{2+}$, but not by $\mathrm{Ca}^{2+}, \mathrm{Ni}^{2+}$, or $\mathrm{Zn}^{2+}$ (Fig. 3e). Similar patterns of divalent cation dependence have previously been seen in the LAGLIDADG homing endonucleases I-DmoI [22], I-CreI [23], and the $\mathrm{HNH}$ homing endonuclease I-HmuI [7]. Endo VII activity was also dependent on $\mathrm{Mg}^{2+}$, which can be replaced by $\mathrm{Mn}^{2+}$ but not by $\mathrm{Ca}^{2+}$ [21]. Furthermore, F-CphI activity was not affected by a low concentration $(5 \mathrm{mM})$ of monovalent ions $\left(\mathrm{Na}^{+}, \mathrm{K}^{+}\right.$, or $\left.\mathrm{NH}_{4}^{+}\right)$, but was inhibited at higher concentrations (Fig. 3d), a
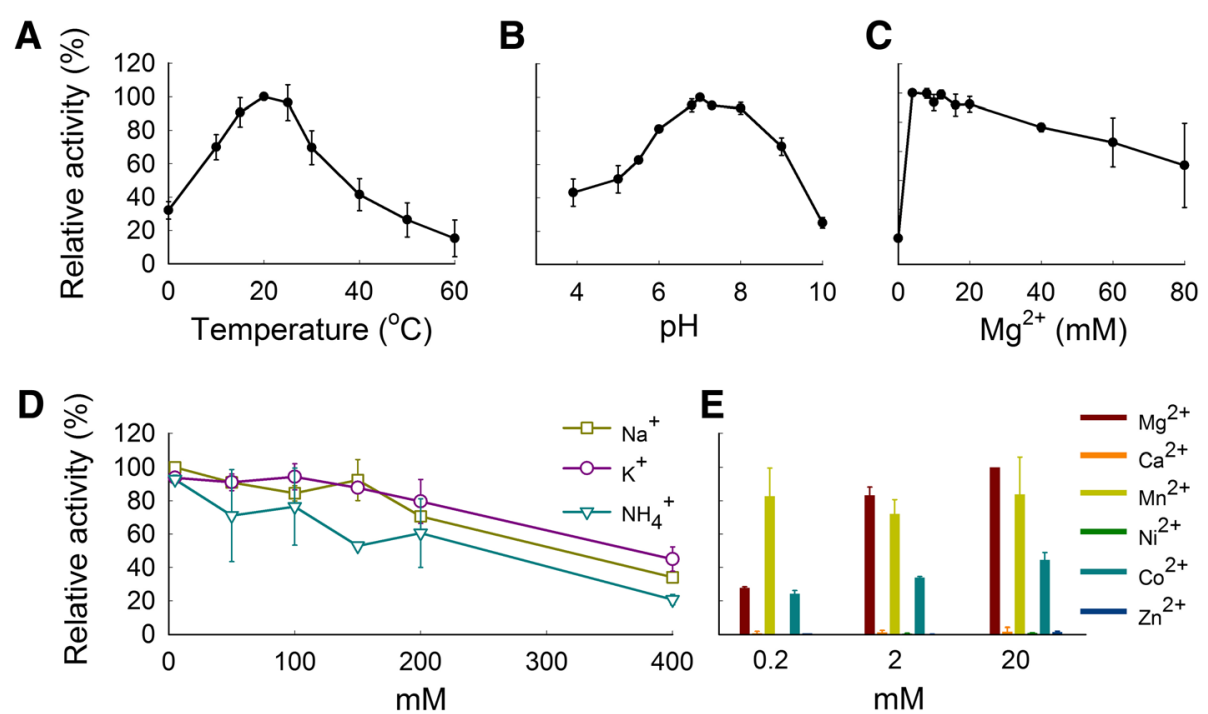

Fig. 3 Optimization of conditions for DNA cleavage by F-Cphl. Purified F-Cphl (1600 nM) was used to digest plasmid DNA (5 nM) in different conditions for $30 \mathrm{~min}$. The graphs show F-Cphl endonuclease activity at different temperatures $\mathbf{a}, \mathrm{pH} \mathbf{b}, \mathrm{Mg}^{2+}$ concentrations $\mathbf{c}$, monovalent cation concentrations $\mathbf{d}$ and divalent cation concentrations e F-Cphl endonuclease activity was calculated by expressing intensities of the linear products relative to the total plasmid DNA substrates. The highest activity for each variable was assigned a value of $100 \%$. Error bars indicate the s.d. from two $\mathbf{b}, \mathbf{d}$ and $\mathbf{e}$ or three $\mathbf{a}$ and $\mathbf{c}$ experiments 
characteristic shared by homing endonucleases [24] and Endo VII [21].

\section{The catalytic domain of T4 endonuclease VII is conserved in $\mathrm{F}-\mathrm{Cphl}$}

The C-terminal part of F-CphI (residues 71-129) aligns with the N-terminal part of Endo VII (residues 10-73) (Fig. 1a and b), which is the catalytic domain of Endo VII $[25,26]$. Using the putative C-terminal catalytic domain of F-CphI to do PSI-BLAST search, a total of 313 sequences were found in sequenced genomes (Additional file 2: Table S1). Multiple sequence alignment of these sequences (Additional file 3: Figure S2) revealed a highly conserved pattern of a central DHDH flanked by an N-terminal $\mathrm{CX}_{2-4} \mathrm{C}$ and a C-terminal $\mathrm{CX}_{2} \mathrm{C}$ (Fig. 1c). The first histidine in the $\mathrm{DHDH}$ region (H41 of Endo VII) was conserved in all the sequences (Additional file 3: Figure S2). The first aspartic acid (D40 of Endo VII) and the second histidine (H43 of Endo VII) were conserved in most of the sequences (Additional file 3: Figure S2). The arginine residue (R54 of Endo VII) after the DHDH sequence was conserved in all the sequences (Fig. 1c, Additional file 3: Figure S2). The asparagine (N62 of Endo VII) distal to $\mathrm{CX}_{2} \mathrm{C}$ was conserved in most sequences (replaced by $\mathrm{R}$ in one sequence Strvi_0243) (Fig. 1c, Additional file 3: Figure S2).

The catalytic domain of T4 Endo VII has been studied extensively. The $\mathrm{CX}_{2-4} \mathrm{C}$ and $\mathrm{CX}_{2} \mathrm{C}$ sequences have been shown to coordinate one atom of zinc in Endo VII [27]. D40 and N62 are the metal ion binding residues in the active site of T4 Endo VII [25, 28]. H41 and H43 are catalytically important in Endo VII [25, 26]. Although the second aspartic acid (D42) in the DHDH region was conserved in many sequences, it is not essential for Endo VII [29]. R54 was shown to be within the active site of T4 Endo VII and near the metal ion [26], but its function is still unknown. The fact that the catalytically important residues for Endo VII were all conserved in
F-CphI (Fig. 1b) suggested to us that they may play similar roles in F-CphI.

\section{Site-directed mutagenesis confirms that the conserved Endo VII residues are also essential for F-Cphl}

Site-directed mutagenesis has been done on T4 Endo VII. C23S and C61S mutants were inactive and they cannot bind to the DNA substrate [27]. C26S and C58S mutants were still active, but when both cysteines were mutated the mutant was inactive and failed to bind to the DNA substrate [27]. D40N, D40A, H41T, H43T [29], and N62D [26] were inactive but they can bind to the DNA substrate, suggesting that these residues are important for catalytic activity. To test whether the essential residues of Endo VII are also important for F-CphI activity, we carried out site-directed mutagenesis on F-CphI at the corresponding residues to Endo VII (C84S, D101N, H102T, N118A, and N118D). No mutation had been made on the conserved arginine of T4 Endo VII, but since it is highly conserved and is near other catalytically important residues, the arginine on F-CphI was also mutated (R110A and R110T) to explore its function.

After expression and purification, the F-CphI mutants had similar soluble protein yields as that of the wild type (Additional file 1: Figure S1B), suggesting that mutations may not disrupt the general folding of the protein. A plasmid containing the $\mathrm{F}-\mathrm{CphI}$ recognition site was used to conduct endonuclease assays to compare the activities of F-CphI wild type and mutants. The closed-circular plasmid DNA was linearized by $400 \mathrm{nM}$ wild type protein, however, cleavage products were not obviously seen for all the mutants, even at $\sim 10$-fold excess protein concentration (Fig. 4). To analyze the cause for abolished DNA cleavage observed with the F-CphI mutants, the DNA binding property was assayed by electrophoretic mobility shift assay using a ${ }^{32}$ P-labeled 60 -bp oligonucleotide containing the F-CphI recognition site. Among the mutant proteins, only D101N and H102T can bind DNA (Additional file 4: Figure S3A), but their DNA binding

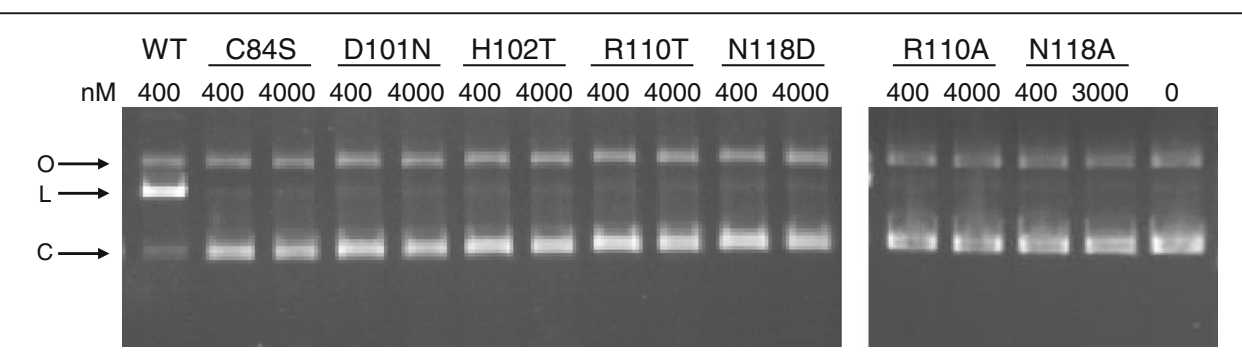

Fig. 4 Activity comparison of F-Cphl wild type and mutants. F-Cphl wild type (400 nM) and mutants (400 nM and 3000 nM for N118A; 400 nM and $4000 \mathrm{nM}$ for others) were incubated with $5 \mathrm{nM}$ closed-circular plasmid DNA (C) at $25^{\circ} \mathrm{C}$ for $1 \mathrm{~h}$. For N118A, $4000 \mathrm{nM}$ cannot be used since it bound to the DNA substrate non-specifically and showed a smear in the gel (data not shown). Reaction products were separated on a $0.8 \%$ agarose gel. Positions of linear $(\mathrm{L})$ and open circular $(\mathrm{O})$ products are shown. DNA substrate without protein added was run in the last lane 
affinities were lower than that of the wild type as shown by their higher Kd values (Additional file 4: Figure S3C). Using an unlabeled non-specific 41-bp DNA complex as the competitor, competition assays confirmed that the binding of the wild type, D101N and H102T to DNA substrate was specific (Additional file 4: Figure S3B). In summary, similar to T4 Endo VII [29], our mutagenesis studies suggested that C84 of F-CphI is important for DNA binding, while D101 and H102 are essential for DNA cleavage. The difference is that F-CphI mutant N118D cannot bind to DNA, while the corresponding Endo VII mutant N62D can bind to DNA [26]. In addition, our results showed that R110 of F-CphI is an essential residue for DNA binding, but it is not clear whether it is also essential for DNA cleavage (see Discussion).

\section{Phylogenetic analysis of proteins containing the Endo VII motif}

Based on the multiple sequence alignment (Additional file 3: Figure S2), we built a phylogenetic tree using the protein sequences that contain the Endo VII motif. The phylogenetic tree consisted of two major branches (Fig. 5). The bottom portion of the tree contained T4 Endo VII (sequence name underlined) and sequences that aligned their whole length with Endo VII (Fig. 5, Additional file 2: Table S1). Moreover, most sequences in the bottom portion of the tree had the Endo VII motif on their N-termini (Fig. 5), which is similar to the domain arrangement of T4. Endo VII. The top portion of the tree contained F-CphI (sequence name underlined) and proteins that aligned the whole length with F-CphI (Fig. 5, Additional file 2: Table S1). Interestingly, most sequences in the top portion of the tree had the Endo VII motif on their C-termini (Fig. 5), similar to the domain arrangement of F-CphI. Our further analysis shown below suggested that sequences in the bottom portion of the tree might be resolvases and those in the top portion might be endonucleases, and hence we named them resolvase and endonuclease clades, respectively.

In the resolvase clade, sequences from myoviruses including T4 Endo VII clustered together (Fig. 5) and they are likely to be the Holliday junction resolvases of these phages. Many sequences from podoviruses also clustered together (Fig. 5). Interestingly, while phage T7 uses endonuclease I as its Holliday junction resolvase, none of these podoviruses encodes an endonuclease I homolog. Therefore, these podoviruses might use Endo VII as their Holliday junction resolvase. The resolvase clade also contained four sequences from putative prophage fragments in bacterial genomes (BTI_1591, BDB_ mp60439, EH105704_05_00150, P845_01269) (Fig. 5). In these bacterial genomes, the Endo VII motif-containing sequences were often adjacent to integrase genes, portal protein genes, or putative phage genes, and thus they were probably resolvases of prophages. Furthermore, we identified 15 sequences from Caenorhabditis (Fig. 5) that were annotated as DNA polymerase B. These proteins are of different lengths, and the Endo VII motifs are in different positions in these proteins (Additional file 2: Table S1). The function of the Endo VII motif in DNA polymerase B remained unknown.

In the endonuclease clade, the podoviruses (Fig. 5) all encode the T7 resolvase endonuclease I, and thus the Endo VII motif-containing proteins might not be resolvases. Moreover, sequences from Yersinia phage Berlin, Yersinia phage YpP-G, and Pseudomonas phage LUZ24 are embedded in a group I intron in the DNA polymerase gene (Intron-encoded, Fig. 5), indicating that they are homing endonucleases [30]. Self-splicing group I introns have also been identified in the DNA polymerase genes of several T7-like phages [31,32]. There are six sequences from myoviruses (Fig. 5). Four of them are intron-encoded (Fig. 5, sequences from Escherichia phage JH2, Escherichia phage EC6, Salmonella phage FSL SP-010, and Escherichia phage 2 JES-2013), while $\mathrm{F}-\mathrm{CphI}$ is associated with a group I intron. The only sequence that is not encoded/associated with an intron is gp6 from Escherichia phage vB EcoM-VR7. Since this phage has another Endo VII motif-containing protein (gp81) in the resolvase clade, gp6 is probably a free-standing homing endonuclease. There are 13 sequences from Helicobacter and one of them Hpy99I has been shown to be a type II restriction endonuclease [33]. Similar to Hpy99I, 12 out of the 13 Helicobacter sequences (except for EMH02975) are adjacent to a methylase, which protects bacterial genomes from self-cleavage. Thus, these sequences are likely to be restriction endonucleases.

\section{Discussion}

Multiple sequence alignment of F-CphI, T4 Endo VII, and the other Endo VII motif-containing proteins revealed a different consensus sequence from that of the $\mathrm{HNH}$ family endonucleases (Additional file 5: Figure S4). The HNH family was named based on a conserved pattern of a central asparagine flanked by two histidines at some distance (the second histidine is often replaced by an asparagine) (Additional file 5: Figure S4) [34, 35]. Superposition of the catalytic domains of T4 Endo VII and the $\mathrm{HNH}$ homing endonuclease I-HmuI showed that H41 and N62 of Endo VII correspond to the first and the second histidines of the HNH motif in I-HmuI, respectively [7, 25]. However, Endo VII and F-CphI do not contain the central asparagine that is conserved in the $\mathrm{HNH}$ family endonucleases, and they both contain several additional conserved residues (D40, H43, and R54 in Endo VII) (Additional file 5: Figure S4). The consensus sequence of the Endo VII motif-containing proteins are 


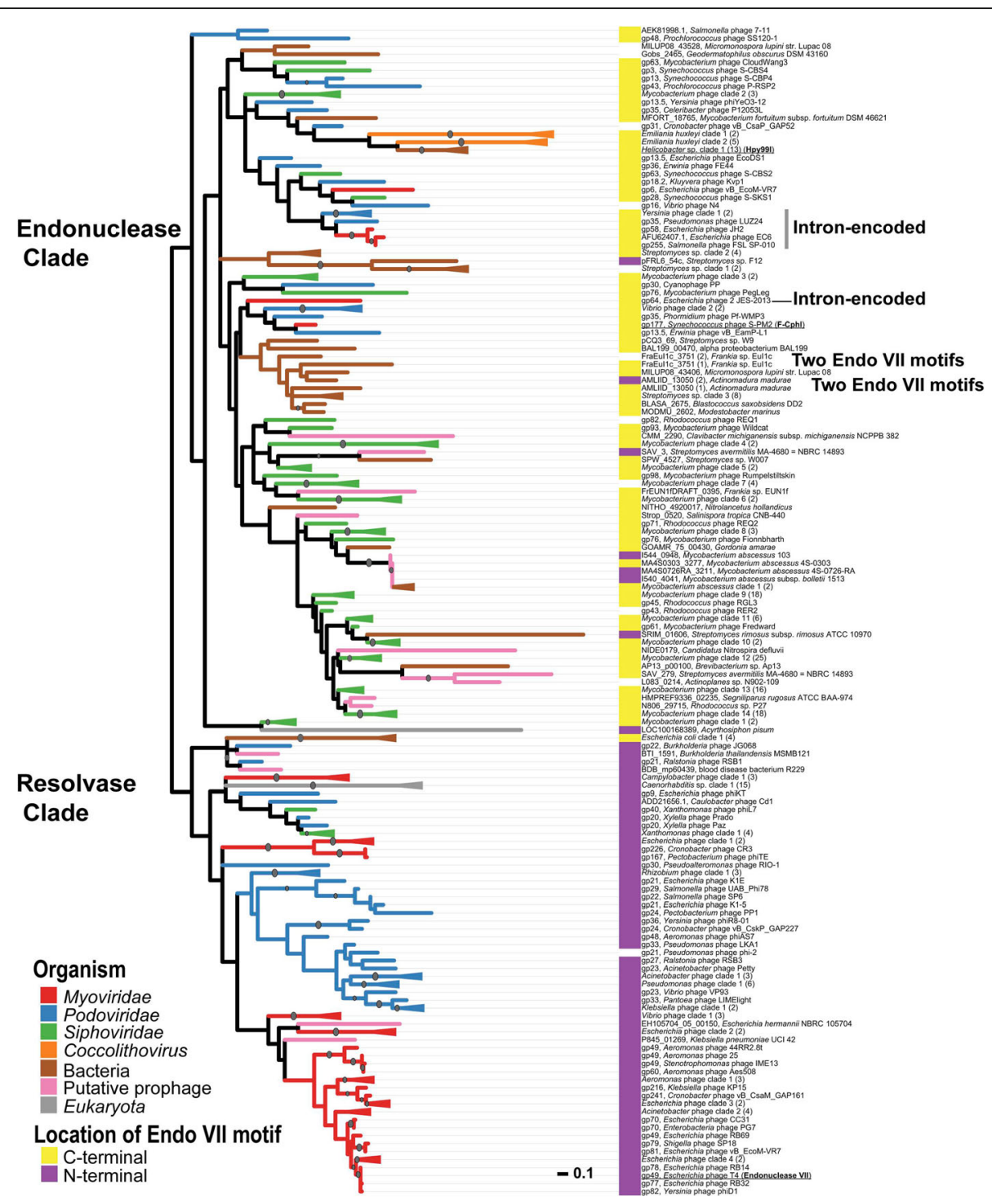

Fig. 5 Phylogenetic tree of the Endo VII motif-containing sequences. An unrooted radial tree was drawn, as there was not a suitable outgroup. The top portion of the tree was named Endonuclease Clade and the bottom portion was named Resolvase Clade. The branch length represents the expected number of substitutions per site, and the scale bar shows 0.1 substitution per site. The branch color shows the organism type. A color bar on the left of a sequence name indicates the location of the Endo VII motif in that sequence, yellow for C-terminal and purple for $\mathrm{N}$-terminal. Dots on the branches indicate bootstrap values $>50 \%$. Branches with triangles on the end contain more than one sequences from the same organism type whose Endo VII motifs are in similar locations of a protein. The number in the parenthesis after a clade name indicates the number of sequences in that clade and the sequences are listed in Additional file 7: Table S2

also different from that of the His-Cys box family homing endonucleases (Additional file 5: Figure S4). Therefore, we proposed that F-CphI represents a new homing endonuclease family and we named it the DHHRN family based on the conserved residues.

Among the DHHRN endonucleases, the crystal structures of T4 Endo VII [28] and Hpy99I [36] have been solved together with their DNA substrates. Although Endo VII is a Holliday junction DNA resolvase and Hpy99I is a restriction endonuclease, their endonuclease domain structures are almost identical and are also similar to those of the HNH family homing endonuclease I-HmuI and the His-Cys box family homing endonuclease I-PpoI (Fig. 6) [36]. Together with the $\mathrm{HNH}$ and His-Cys box families [11], the DHHRN family also belongs to the $\beta \beta \alpha$-metal endonuclease superfamily.

Our mutagenesis experiments suggested that D101 and H102 were catalytically important for F-CphI. The corresponding residues are not only conserved in Endo VII and Hpy99I, they are also within the active site and have catalytic functions $[28,36]$. Thus, the active site structure of F-CphI is likely to be similar to those of 

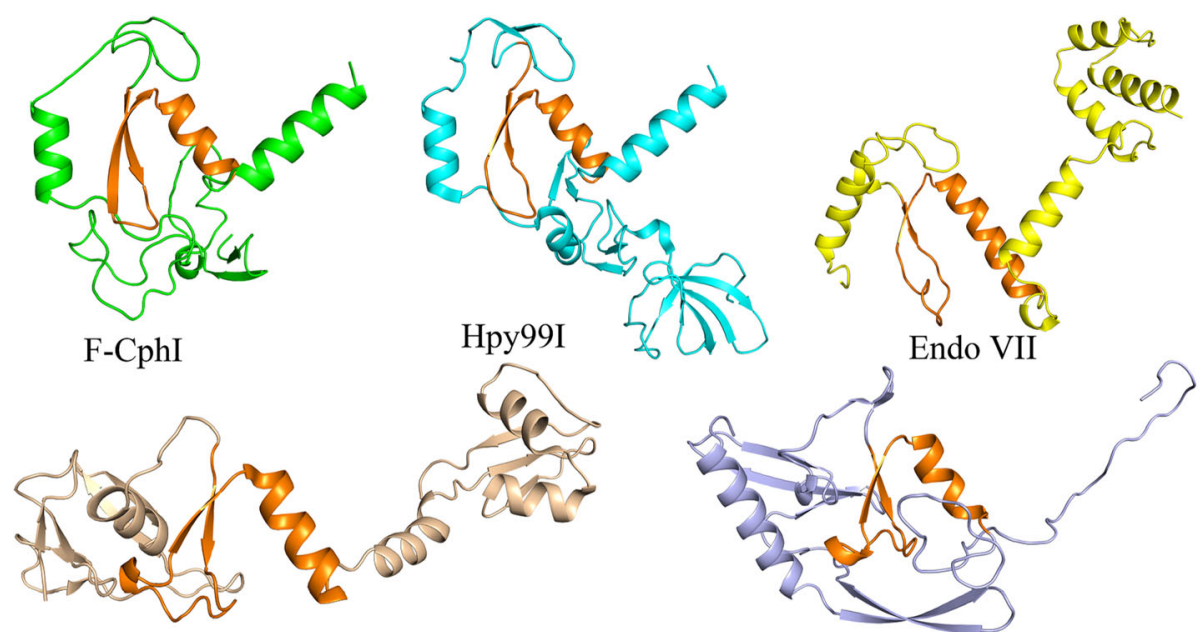

I-HmuI

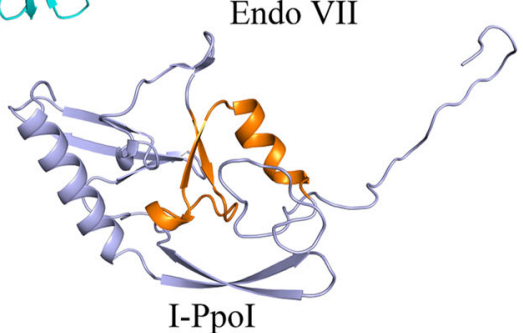

Fig. 6 Structures of the Bßa-metal superfamily endonucleases. Protein structures of Hpy99I [36], Endo VII [26], I-Hmul [7], and I-Ppol [5] were obtained from previous studies. The structure model of F-Cphl was generated based on Hpy99l structure using the online tool SWISS-MODEL (www.swissmodel.expasy.org/). The conserved active-site motif of each protein was colored orange and presented in the same orientation

Endo VII and Hpy99I. Indeed, we used Hpy99I structure to model the structure of F-CphI and found that their core structures harboring the active site are almost identical (Fig. 6). Since Hpy99I uses its active site region for both DNA cleavage and binding [36], F-CphI may also use its active site region for DNA binding. Furthermore, our mutagenesis experiments showed that R110 and N118 affected DNA binding of F-CphI, while the corresponding residues are shown in Hpy99I to have catalytic functions [36]. Thus, we proposed that R110 and N118 of F-CphI may play a role in both DNA binding and cleavage.

It has been shown that the catalytic motifs of LAGLIDADG, GIY-YIG, HNH, His-Cys box, PD-(D/E)$\mathrm{XK}$, and EDxHD homing endonucleases are used by proteins of a variety of functions. These proteins include non-specific DNA-degradation colicins, restriction endonucleases, DNA repair enzymes, Holliday junction resolvases, intron-splicing factors, and transcription factors [4]. It is still not clear whether these proteins diverged from a common ancestor or the homing endonucleases were modified by the host organisms to be specialized in other cellular functions [4]. Similar to the six established homing endonuclease families, our phylogenetic analysis (Fig. 5) showed that the DHHRN motif can be used by homing endonucleases, Holliday junction resolvases, restriction endonucleases, and possibly DNA polymerases. Our analysis facilitates the functional prediction of many previously unknown proteins.

\section{Conclusions}

The catalytically important residues of T4 Endo VII are all conserved and essential for F-CphI activity. F-CphI represents a new homing endonuclease family, the DHHRN family. Our phylogenetic analysis showed that the DHHRN family proteins include homing endonucleases, Holliday junction resolvases, restriction endonucleases, and possibly DNA polymerases.

\section{Methods}

\section{Cloning of $\mathrm{F}-\mathrm{Cphl}$ gene and site-directed mutagenesis}

F-CphI wild type and mutants were cloned into pBAD/ $M y c$-HisB plasmid (Invitrogen), in which protein expression is tightly controlled by the $\mathrm{P}_{\mathrm{BAD}}$ promoter and can be induced by L-arabinose. Primers CyaU-Nco and CyaD-Xba were used to amplify the F-CphI gene from S-PM2 phage. PCR was carried out with $0.02 \mathrm{U} / \mu \mathrm{l}$ KOD HiFi DNA polymerase (Novagen) in $120 \mathrm{mM}$ Tris- $\mathrm{HCl}(\mathrm{pH} 8.0), 10 \mathrm{mM}$ $\mathrm{KCl}, 6 \mathrm{mM}\left(\mathrm{NH}_{4}\right)_{2} \mathrm{SO}_{4}, 0.1 \%$ Triton X-100, 0.001\% BSA, $1 \mathrm{mM} \mathrm{MgCl}, 0.2 \mathrm{mM} \mathrm{dNTPs}$, and $0.4 \mu \mathrm{M}$ of each primer. PCR cycling conditions consisted of a hot start at $94{ }^{\circ} \mathrm{C}$ for 5 min, followed by 25 cycles $\left(98{ }^{\circ} \mathrm{C}\right.$ for $15 \mathrm{~s}, 50{ }^{\circ} \mathrm{C}$ for $2 \mathrm{~s}$ and $74{ }^{\circ} \mathrm{C}$ for $20 \mathrm{~s}$ ), followed by incubation at $74{ }^{\circ} \mathrm{C}$ for $7 \mathrm{~min}$. Site-directed mutagenesis was performed by a PCR based overlap extension method [37]. PCR products were inserted into the NcoI and XbaI sites of the pBAD/ $M y c$-HisB vector and transformed into E. coli Top10 cells. Primer CyaD-Xba does not have a stop codon and hence the cloned genes are in frame with the downstream His-tag. The desired mutations were confirmed by sequencing. Oligonucleotides are listed in Additional file 6: Table S3.

\section{Protein expression and purification}

Strains for expressing F-CphI wild type and mutants were grown at $37{ }^{\circ} \mathrm{C}$ overnight in the $\mathrm{LB}$ medium 
containing $50 \mu \mathrm{g} / \mathrm{ml}$ ampicillin. Overnight cultures were diluted 1:100 with the same medium, grown at $37{ }^{\circ} \mathrm{C}$ until $\mathrm{OD}_{600}=0.5$, and then used for protein induction. The concentration of inducer (L-arabinose), the induction time, and temperature were optimized. Maximum protein expression was induced by $0.02 \% \mathrm{~L}$-arabinose (final concentration) at $16{ }^{\circ} \mathrm{C}$ for $24 \mathrm{~h}$. Bacterial cells were harvested by centrifugation at $6000 \mathrm{~g}$ for $20 \mathrm{~min}$. The cell pellet was suspended with the binding buffer (20 mM sodium phosphate, $500 \mathrm{mM} \mathrm{NaCl}, 20 \mathrm{mM}$ imidazole, $\mathrm{pH}$ 7.4) and disrupted by sonication. The crude lysate was centrifuged at 10,000 g for $20 \mathrm{~min}$ and the supernate was loaded on a HisTrap FF crude column (GE Healthcare). Protein purification was performed according to the manufacturer's instructions. Protein was eluted from the column with the elution buffer $(20 \mathrm{mM}$ sodium phosphate, $500 \mathrm{mM} \mathrm{NaCl}, 500 \mathrm{mM}$ imidazole, $\mathrm{pH}$ 7.4) and stored in $50 \%$ glycerol at $-20^{\circ} \mathrm{C}$.

\section{Endonuclease assay with plasmid substrate}

The plasmid pSBM4-TA [12] containing the phage S-BM4 $p s b A$ gene was used as substrate for F-CphI. $5 \mathrm{nM}$ plasmid was incubated with $1600 \mathrm{nM}$ purified F-CphI in the standard endonuclease assay buffer $(50 \mathrm{mM}$ Tris- $\mathrm{HCl}, \mathrm{pH} 7.5$, $50 \mathrm{mM} \mathrm{NaCl}, 10 \mathrm{mM} \mathrm{MgCl} 2$ and $0.1 \mathrm{mg} / \mathrm{ml} \mathrm{BSA}$ ) at $25{ }^{\circ} \mathrm{C}$ for $30 \mathrm{~min}$ when the reaction was still linear. The reaction was stopped by adding $1 \mu \mathrm{l} 10 \times$ endonuclease stop buffer (100 mM Tris-HCl, 25 mM EDTA, 5\% SDS, $\mathrm{pH}$ 7.5) and kept on ice. Reaction products were separated in a $0.8 \%$ agarose gel and stained with EtBr. To determine the optimal conditions for F-CphI, each parameter in the standard endonuclease buffer was changed while others remained the same. Formate (for $\mathrm{pH}$ 3.9), succinic acid (for $\mathrm{pH}$ 5.0, 5.4 and 6.0), PIPES (for $\mathrm{pH} 6.8$, 7.0 and 7.3), Tris (for $\mathrm{pH} 8.0$ ), and CHES (for $\mathrm{pH} 9.0$ and 10.0) were used to prepare different $\mathrm{pH}$ buffers and their final concentrations were all $50 \mathrm{mM}$. The optimal endonuclease assay buffer was characterized as $50 \mathrm{mM}$ PIPES (pH 7.0), $5 \mathrm{mM} \mathrm{MgCl}, 50 \mathrm{mM} \mathrm{KCl}$ and $0.1 \mathrm{mg} / \mathrm{ml} \mathrm{BSA}$. To compare the activities of F-CphI wild type and mutants, $5 \mathrm{nM}$ pSBM4-TA plasmid was incubated with different amounts of proteins at $25^{\circ} \mathrm{C}$ for $1 \mathrm{~h}$ in the optimal endonuclease assay buffer.

\section{Endonuclease assay with ${ }^{32} \mathrm{P}$-labeled substrate}

Oligonucleotide duplex containing the F-CphI cleavage site [12] was generated by annealing complementary oligonucleotides SBM4-60Top and SBM4-60Top-r at $90{ }^{\circ} \mathrm{C}$ for $5 \mathrm{~min}$ and cooling to $55{ }^{\circ} \mathrm{C}$ in $25 \mathrm{mM}$ Tris/ $\mathrm{HCl}$ (pH 8.0) and $50 \mathrm{mM} \mathrm{NaCl}$. Individually $5^{\prime}$ end-labeled targets were generated by labeling one of the oligonucleotides on its $5^{\prime}$ end (with $\left[\gamma^{-32} \mathrm{P}\right] \mathrm{ATP}$ and T4 polynucleotide kinase) before being used in annealing reactions with an unlabeled partner. Labeled duplexes were purified using a QIAquick PCR Purification Kit (Qiagen). $10 \mathrm{nM}$ labeled duplex was incubated with $1 \mu \mathrm{M} F-C p h I$ in the optimal endonuclease assay buffer at $25{ }^{\circ} \mathrm{C}$. Reaction products were extracted with an equal volume of phenol and separated on a $4 \%$ denaturing polyacrylamide gel. Sequences of SBM4-60Top and SBM4-60Top-r are listed in Additional file 6: Table S3.

\section{Electrophoretic mobility shift assay}

To compare the DNA binding ability of F-CphI wild type and mutants, both specific and nonspecific DNA duplexes were used. A specific oligonucleotide duplex containing the F-CphI cleavage site was generated by annealing $5^{\prime}$ end labeled SBM4-60Top and unlabeled SBM4-60Top-r. Nonspecific competitor duplex was generated using oligonucleotide endoV41A and its complementary sequence endoV41comp (Additional file 6: Table S3). For assays without competitor, $2 \mathrm{nM}$ labeled SBM4-60Top duplex was incubated on ice for $15 \mathrm{~min}$ with different amounts of protein in $10 \mathrm{mM}$ Tris- $\mathrm{HCl}$ (pH 8.0), $2 \%$ glycerol, $2 \mu \mathrm{g} / \mathrm{ml}$ poly-dIdC, $2 \mu \mathrm{g} / \mathrm{ml} \mathrm{BSA}$ and $0.2 \mathrm{mM}$ DTT in total $20 \mu \mathrm{l}$ reaction volume. For assays with nonspecific competitor, $2 \mathrm{nM}$ labeled SBM460 Top duplex and $200 \mathrm{nM}$ protein were incubated with different amounts of endoV41A duplex. The free DNA and protein-bound complexes were separated on $8 \%$ native polyacrylamide gel with $1 \mathrm{X}$ TBE buffer $(89 \mathrm{mM}$ Tris, 2 mM EDTA, 89 mM Boric acid, $\mathrm{pH}$ 8.3).

\section{Binding affinities of F-Cphl wild type and mutants to DNA substrates}

Quantitation of band intensities from the electrophoretic mobility shift assay were performed in the GelQuant. NET software (biochemlabsolutions.com). Band intensity of DNA-bound complex at protein concentration of $0 \mathrm{nM}$ was used as the background signal, and was subtracted from the signal intensities obtained from all the bands. The fraction of DNA bound was thus calculated from bound/(bound + unbound), and was plotted versus the concentrations of F-CphI wild type, and D101N and H102T mutants, respectively. The data were then fitted by non-linear regression model with the nlsLM function of minpack. $\mathrm{lm}$ package in $\mathrm{R}$, using the following equation modified from previous studies [38, 39]:

$$
\mathrm{F}=\mathrm{A} \times \mathrm{P}_{0} /\left(\mathrm{Kd}+\mathrm{P}_{0}\right)
$$

where $F$ is the fraction of DNA bound, $A$ is the maximal fraction of DNA bound, $\mathrm{P}_{0}$ is the concentration of total protein, and $\mathrm{Kd}$ is the apparent equilibrium dissociation constant.

\section{Homolog search}

In-house BLAST searches were carried out against the downloaded non-redundant (nr) protein database (http://www.ncbi.nlm.nih.gov/BLAST/). In each search, 
we performed BLASTp and PSI-BLAST with two iterations and set a final $e$-value cut-off of 1E-4. In search of the Endo VII homologs, both the catalytic domain of T4 Endo VII (residues 1-97) and the corresponding sequence of F-CphI (residues 62-139) were used as the query protein sequences. The resulting 313 protein sequences from complete genomes were fetched from NCBI for further analysis. In search of the $\mathrm{HNH}$ and His-Cys box family proteins, the catalytic domains of I-Hmul (residues 49-97) from Bacillus phage SP01 and I-PpoI (residues 93-127) from Physarum polycephalum were used as the query sequences, respectively. Sequences from incomplete genomes or obtained from environmental samples were discarded prior to protein sequence extraction.

\section{Multiple sequence alignment}

MAFFT [40] and Clustal X [41] were used for multiple sequence alignment of the homologous sequences. The resulting alignment was then manually refined through editing in Alignment Explorer of MEGA6 [42]. The final alignment, $133 \mathrm{bp}$ in length with gaps, represented the most conserved sequence blocks across all compared organisms. The alignment was then analyzed on ESPript (http://espript.ibcp.fr/) to obtain the consensus sequence with conserved amino acids highlighted.

\section{Sequence logo}

In order to profile the consensus sequence from the multiple sequence alignment, we generated sequence logo on the Skylign website (http://skylign.org), which is based on the profile hidden Markov model (HMM) analysis [43]. Profile HMM establishes a position-specific scoring sequence profile representing a multiple sequence alignment [43]. Skylign creates a graphical sequence logo (HMM logo) from the sequence alignment, which not only represents the extent of conservation at each position, but also shows the probabilities of a profile HMM [44]. In the HMM logo, the height of a stack of letters at each position shows the information content in bits, which represents the extent to which the position-specific distribution of letters differs from that of the background. At each position, the height of a letter relative to the total height of the stack of letters correlates to a particular letter's frequency at that position [44].

\section{Phylogenetic analysis}

ProtTest [45] was used with default parameters to select the best amino acid substitution model. PhyML [46] was used for phylogenetic analysis of the Endo VII domain homologs, based on the maximum-likelihood principle. The parameters for the command-line PhyML program were set as: - $d$ aa -b 100 -m Blosum62 -f m -v e -s SPR -o tlr. The output phylogenetic tree was demonstrated via iTOL (http://itol.embl.de).

\section{Additional files}

\begin{abstract}
Additional file 1: Figure S1. Purification of F-Cphl wild type and mutants. (A) E. coli cells were induced to express F-Cphl and then disrupted by sonication. The crude lysate was centrifuged and the supernate was loaded on a HisTrap FF crude column. Five elutions (1 ml each) were collected. The crude lysate, supernate, pellet, and five elutions were separated on 15\% SDS-PAGE. (B) Purified F-Cphl wild type and mutants (second elutions) were separated on 15\% SDS-PAGE. (PDF $233 \mathrm{~kb}$ )
\end{abstract}

Additional file 2: Table S1. Endo VII motif-containing proteins. Proteins containing the Endo VII motif were revealed by PSI-BLAST searches. In the last column, the position of the Endo VII motif in a protein is shown. The Endo VII motif of a protein could be in the $\mathrm{C}$ terminus (C), the $\mathrm{N}$ terminus $(\mathrm{N}$ ), or in the middle (indicated by residue numbers/full length of a protein). In the same column, F-Cphl and Endo VII indicate that a protein aligns with the full length of F-Cphl or Endo VII, respectively. (XLSX $29 \mathrm{~kb})$

Additional file 3: Figure S2. Multiple sequence alignment of the Endo VII motif sequences. Dots represent gaps in the alignment. Totally conserved residues at a position are shown by white letters in red background. Residues with conservation above $70 \%$ at a position are shown by red letters in blue boxes. The position numbers of F-Cphl and Endo VII residues are shown on top of the alignment. (PDF $1993 \mathrm{~kb}$ )

Additional file 4: Figure S3. Gel shift assay of F-Cphl wild type and mutants. (A) $2 \mathrm{nM}^{32}$ P labeled 60 bp duplex containing the F-Cphl recognition site was incubated on ice for 15 min with increasing concentration of each protein. The free DNA (F) and protein-bound complexes (C) were separated on $8 \%$ native polyacrylamide gel. (B) 2 nM ${ }^{32} \mathrm{P}$ labeled 60 bp duplex containing the $\mathrm{F}-\mathrm{Cphl}$ recognition site and $200 \mathrm{nM}$ protein were incubated with increasing concentration of unlabeled non-specific $60 \mathrm{bp}$ duplex (0 nM to $20 \mathrm{nM}$ ). The first lane in each gel shows the pattern of free DNA (no protein was added in the reaction). (C) Using the gels in $\mathbf{A}$, the fractions of protein-bound complexes for wild type, D101N, and H102T were plotted against protein concentrations. The binding curves were generated from the non-linear regression fitted data, and were used to estimate the apparent equilibrium dissociation constant (Kd). Kd values are shown in each graph and errors represent 95\% confidence interval. (PDF $126 \mathrm{~kb}$ )

Additional file 5: Figure S4. Sequence logos of the DHHRN, HNH, and His-Cys box endonuclease families. Sequences near the active site of an endonuclease family are used to generate sequence logos. At each position of the sequence logo, the total height of a stack of letters shows the information content in bits that is calculated from a profile hidden Markov model, and the height of a letter relative to the total height of letters at a position represents the letter's frequency. The red lines indicate gaps in the multiple sequence alignment. Above each sequence logo, the corresponding residue numbers of Endo VII, I-Hmul, and I-Ppol are shown, which are representatives of the DHHRN, HNH, and His-Cys box families, respectively. Black boxes show the corresponding catalytic residues used by Endo VII, I-Hmul, and I-Ppol. (PDF $351 \mathrm{~kb}$ )

Additional file 6: Table S3. Oligonucleotides used in this study (restriction sites are underlined and mutated sites are italicized). (DOCX $12 \mathrm{~kb}$ )

Additional file 7: Table S2. Clades of Endo VII motif-containing proteins as shown in Fig. 5. (XLSX $17 \mathrm{~kb})$

Abbreviations

Endo VII: Endonuclease VII; IIS: Intron insertion site; ORF: Open reading frames

Acknowledgements

We thank David Shub, Ning Gao, Xinquan Wang, and Yinan Jiang for helpful discussions.

\section{Funding}

This study is supported by grants to Qinglu Zeng from the National Natural Science Foundation of China (Project number 41476147) and the Research Grants Council of the Hong Kong Special Administrative Region, China (Project numbers 16103414 and 689813). 


\section{Availability of data and materials}

The datasets analysed during the current study are available in the non-redundant (nr) protein database (http://www.ncbi.nlm.nih.gov/BLAST/) and the information of the protein sequences is listed in Additional file 2: Table S1. The materials used during the current study are available from the corresponding author on reasonable request.

\section{Authors' contributions}

XF did bioinformatics analysis. Y $\sqcup$ modeled the structure of F-Cphl. KL generated plasmids for F-Cphl and mutant protein expression. QZ did endonuclease assays. XF and QZ wrote the manuscript. All authors read and approved the final manuscript.

\section{Ethics approval and consent to participate}

Not applicable.

\section{Consent for publication}

Not applicable.

\section{Competing interests}

The authors declare that they have no competing interests.

\section{Publisher's Note}

Springer Nature remains neutral with regard to jurisdictional claims in published maps and institutional affiliations.

\section{Author details}

'Department of Ocean Science, The Hong Kong University of Science and Technology, Clear Water Bay, Kowloon, Hong Kong, China. ${ }^{2}$ School of Life Sciences, University of Science and Technology of China, Hefei 230027, Anhui, China. ${ }^{3}$ Division of Life Science, The Hong Kong University of Science and Technology, Clear Water Bay, Kowloon, Hong Kong, China. ${ }^{4}$ HKUST Shenzhen Research Institute, Shenzhen, China.

Received: 21 May 2018 Accepted: 31 July 2018

Published online: 09 August 2018

\section{References}

1. Dujon B. Group I introns as mobile genetic elements: facts and mechanistic speculations--a review. Gene. 1989;82(1):91-114.

2. Belle A, Landthaler M, Shub DA. Intronless homing: site-specific endonuclease SegF of bacteriophage T4 mediates localized marker exclusion analogous to homing endonucleases of group I introns. Genes Dev. 2002;16(3):351-62.

3. Liu Q, Belle A, Shub DA, Belfort M, Edgell DR. SegG endonuclease promotes marker exclusion and mediates co-conversion from a distant cleavage site. J Mol Biol. 2003:334(1):13-23.

4. Taylor GK, Stoddard BL. Structural, functional and evolutionary relationships between homing endonucleases and proteins from their host organisms. Nucleic Acids Res. 2012;40(12):5189-200.

5. Flick KE, Jurica MS, Monnat RJ Jr, Stoddard BL. DNA binding and cleavage by the nuclear intron-encoded homing endonuclease I-Ppol. Nature. 1998; 394(6688):96-101.

6. Jurica MS, Monnat RJ Jr, Stoddard BL. DNA recognition and cleavage by the LAGLIDADG homing endonuclease I-Crel. Mol Cell. 1998;2(4):469-76.

7. Shen BW, Landthaler M, Shub DA, Stoddard BL. DNA binding and cleavage by the HNH homing endonuclease I-Hmul. J Mol Biol. 2004;342(1):43-56.

8. Van Roey P, Meehan L, Kowalski JC, Belfort M, Derbyshire V. Catalytic domain structure and hypothesis for function of GIY-YIG intron endonuclease I-Tevl. Nat Struct Biol. 2002:9(11):806-11.

9. Zhao L, Bonocora RP, Shub DA, Stoddard BL. The restriction fold turns to the dark side: a bacterial homing endonuclease with a PD-(D/E)-XK motif. EMBO J. 2007;26(9):2432-42.

10. Taylor GK, Heiter DF, Pietrokovski S, Stoddard BL. Activity, specificity and structure of I-Bth0305I: a representative of a new homing endonuclease family. Nucleic Acids Res. 2011;39(22):9705-19.

11. Kuhlmann UC, Moore GR, James R, Kleanthous C, Hemmings AM. Structural parsimony in endonuclease active sites: should the number of homing endonuclease families be redefined? FEBS Lett. 1999:463(1-2):1-2.

12. Zeng $\mathrm{Q}$, Bonocora RP, Shub DA. A free-standing homing endonuclease targets an intron insertion site in the psbA gene of cyanophages. Curr Biol. 2009;19(3):218-22.
13. Mann NH, Clokie MR, Millard A, Cook A, Wilson WH, Wheatley PJ, Letarov A, Krisch HM. The genome of S-PM2, a "photosynthetic" T4-type bacteriophage that infects marine Synechococcus strains. J Bacteriol. 2005;187(9):3188-200.

14. Millard A, Clokie MR, Shub DA, Mann NH. Genetic organization of the psbAD region in phages infecting marine Synechococcus strains. Proc Natl Acad Sci U S A. 2004;101(30):11007-12.

15. Kemper B, Garabett M, Courage U. Studies on T4-head maturation. 2. Substrate specificity of gene-49-controlled endonuclease. Eur J Biochem. 1981;115(1):133-41.

16. Perrin A, Buckle M, Dujon B. Asymmetrical recognition and activity of the IScel endonuclease on its site and on intron-exon junctions. EMBO J. 1993; 12(7):2939-47.

17. Chu FK, Maley F, Wang AM, Pedersen-Lane J, Maley G. Purification and substrate specificity of a T4 phage intron-encoded endonuclease. Nucleic Acids Res. 1991;19(24):6863-9.

18. Loizos N, Silva GH, Belfort M. Intron-encoded endonuclease I-Tevll binds across the minor groove and induces two distinct conformational changes in its DNA substrate. J Mol Biol. 1996;255(3):412-24.

19. Carter JM, Friedrich NC, Kleinstiver B, Edgell DR. Strand-specific contacts and divalent metal ion regulate double-strand break formation by the GIY-YIG homing endonuclease I-Bmol. J Mol Biol. 2007;374(2):306-21.

20. Elde M, Willassen NP, Johansen S. Functional characterization of isoschizomeric his-Cys box homing endonucleases from Naegleria. Eur J Biochem. 2000;267(24):7257-66.

21. Kemper B, Garabett M. Studies on T4-head maturation. 1. Purification and characterization of gene-49-controlled endonuclease. Eur J Biochem. 1981; 115(1):123-31.

22. Aagaard C, Awayez MJ, Garrett RA. Profile of the DNA recognition site of the archaeal homing endonuclease I-Dmol. Nucleic Acids Res. 1997;25(8): 1523-30.

23. Chevalier B, Sussman D, Otis C, Noel AJ, Turmel M, Lemieux C, Stephens K, Monnat RJ Jr, Stoddard BL. Metal-dependent DNA cleavage mechanism of the I-Crel LAGLIDADG homing endonuclease. Biochemistry. 2004;43(44): 14015-26.

24. Wang J, Kim HH, Yuan X, Herrin DL. Purification, biochemical characterization and protein-DNA interactions of the I-Crel endonuclease produced in Escherichia coli. Nucleic Acids Res. 1997;25(19):3767-76.

25. Raaijmakers H, Toro I, Birkenbihl R, Kemper B, Suck D. Conformational flexibility in T4 endonuclease VII revealed by crystallography: implications for substrate binding and cleavage. J Mol Biol. 2001;308(2):311-23.

26. Raaijmakers H, Vix O, Toro I, Golz S, Kemper B, Suck D. X-ray structure of T4 endonuclease VII: a DNA junction resolvase with a novel fold and unusual domain-swapped dimer architecture. EMBO J. 1999;18(6):1447-58.

27. Giraud-Panis MJ, Duckett DR, Lilley DM. The modular character of a DNA junction-resolving enzyme: a zinc-binding motif in bacteriophage T4 endonuclease VII. J Mol Biol. 1995;252(5):596-610.

28. Biertumpfel C, Yang W, Suck D. Crystal structure of T4 endonuclease VII resolving a Holliday junction. Nature. 2007;449(7162):616-20.

29. Giraud-Panis MJ, Lilley DM. T4 endonuclease VII. Importance of a histidineaspartate cluster within the zinc-binding domain. J Biol Chem. 1996;271(51): 33148-55.

30. Ceyssens PJ, Hertveldt K, Ackermann HW, Noben JP, Demeke M, Volckaert $\mathrm{G}$, Lavigne $\mathrm{R}$. The intron-containing genome of the lytic Pseudomonas phage LUZ24 resembles the temperate phage PaP3. Virology. 2008;377(2): 233-8.

31. Bonocora RP, Shub DA. A self-splicing group I intron in DNA polymerase genes of T7-like bacteriophages. J Bacteriol. 2004;186(23):8153-5.

32. Bonocora RP, Shub DA. A likely pathway for formation of mobile group I introns. Curr Biol. 2009;19(3):223-8.

33. Kong H, Lin LF, Porter N, Stickel S, Byrd D, Posfai J, Roberts RJ. Functional analysis of putative restriction-modification system genes in the Helicobacter pylori 199 genome. Nucleic Acids Res. 2000;28(17):3216-23.

34. Gorbalenya AE. Self-splicing group I and group II introns encode homologous (putative) DNA endonucleases of a new family. Protein Sci. 1994;3(7):1117-20

35. Shub DA, Goodrich-Blair H, Eddy SR. Amino acid sequence motif of group I intron endonucleases is conserved in open reading frames of group II introns. Trends Biochem Sci. 1994;19(10):402-4.

36. Sokolowska M, Czapinska H, Bochtler M. Crystal structure of the beta beta alpha-me type II restriction endonuclease Hpy99| with target DNA. Nucleic Acids Res. 2009;37(11):3799-810. 
37. Horton RM. PCR-mediated recombination and mutagenesis. SOEing together tailor-made genes. Mol Biotechnol. 1995;3(2):93-9.

38. Yakhnin AV, Yakhnin H, Babitzke P. Gel Mobility Shift Assays to Detect Protein-RNA Interactions. In: Keiler K. (eds) Bacterial Regulatory RNA. Methods in Molecular Biology (Methods and Protocols), vol 905. Totowa: Humana Press; 2012

39. Heffler MA, Walters RD, Kugel JF. Using electrophoretic mobility shift assays to measure equilibrium dissociation constants: GAL4-p53 binding DNA as a model system. Biochem Mol Biol Educ. 2012;40(6):383-7.

40. Katoh K, Standley DM. MAFFT multiple sequence alignment software version 7: improvements in performance and usability. Mol Biol Evol. 2013. 30(4):772-80.

41. Larkin MA, Blackshields G, Brown NP, Chenna R, McGettigan PA, McWilliam H, Valentin F, Wallace IM, Wilm A, Lopez R, et al. Clustal W and Clustal X version 2.0. Bioinformatics. 2007:23(21):2947-8.

42. Tamura K, Stecher G, Peterson D, Filipski A, Kumar S. MEGA6: molecular evolutionary genetics analysis version 6.0. Mol Biol Evol. 2013;30(12):2725-9.

43. Eddy SR. Profile hidden Markov models. Bioinformatics (Oxf, Engl). 1998; 14(9):755-63.

44. Wheeler TJ, Clements J, Finn RD. Skylign: a tool for creating informative, interactive logos representing sequence alignments and profile hidden Markov models. BMC bioinformatics. 2014;15(1):7.

45. Darriba D, Taboada GL, Doallo R, Posada D. ProtTest 3: fast selection of bestfit models of protein evolution. Bioinformatics. 2011:27(8):1164-5.

46. Guindon S, Gascuel O, Rannala B. A simple, fast, and accurate algorithm to estimate large phylogenies by maximum likelihood. Syst Biol. 2003;52(5): 696-704.

Ready to submit your research? Choose BMC and benefit from:

- fast, convenient online submission

- thorough peer review by experienced researchers in your field

- rapid publication on acceptance

- support for research data, including large and complex data types

- gold Open Access which fosters wider collaboration and increased citations

- maximum visibility for your research: over $100 \mathrm{M}$ website views per year

At $\mathrm{BMC}$, research is always in progress.

Learn more biomedcentral.com/submissions 